\title{
RESPUESTA DE LA REGENERACIÓN NATURAL DE LA Uncaria tomentosa (Willd) D.C. “UÑA DE GATO”, AL EFECTO DE LA LUZ EN ECOSISTEMAS BOSCOSOS PRIMARIOS INTERVENIDOS DENTRO DEL BOSQUE NACIONAL ALEXANDER VON HUMBOLDT, PUCALLPA - PERÚ
}

\section{THE EFFECT OF LIGHT ON THE NATURAL REGENERATION OF Uncaria tomentosa (Willd) DC "CAT'S CLAW", IN A FOREST ECOSYSTEM AT THE “BOSQUE NACIONAL ALEXANDER VON HUMBOLDT”, PUCALLPA - PERU}

\author{
Ayling Wetzell Canales-Springett ${ }^{1}$, Aldo Ceroni Stuva ${ }^{2}$, Gilberto Domínguez Torrejón ${ }^{3}$ y Andrés Castillo \\ Quiliano $^{4}$
}

\begin{abstract}
Resumen
La presente investigación fue llevada a cabo en el Bosque Nacional Alexander Von HumboldtBNAVH, ubicado en la jurisdicción del distrito de Irazola, Provincia de Padre Abad y los distritos de Tournavista y Puerto Inca, Provincia de Puerto Inca, Regiones de Ucayali y Huánuco respectivamente. El objetivo fue evaluar los factores climáticos, específicamente iluminación, que influyen en la regeneración natural de Uncaria tomentosa (Willd.) D.C (Uña de gato) en bosques naturales primarios intervenidos dentro del BNAVH. La evaluación, se realizó en seis tipos de bosques primarios intervenidos y caracterizados por la apertura del dosel superior por efecto del ancho de las viales de extracción de madera y su orientación con respecto a la dirección de iluminación solar, dentro de los cuales se establecieron parcelas semipermanentes de evaluación. En cada una de ellas se midieron variables biológicas de Uncaria respecto al factor iluminación que llega al sotobosque. Se encontró que en estos tipos de bosque, se pueden encontrar en promedio más de 900 individuos por ha. de regeneración natural en buenas condiciones fitosanitarias y con diámetros promedio de $14 \mathrm{~mm}$. Respecto al tamaño de planta se observó que 211 individuos se encontraban en el rango de 30 a $90 \mathrm{~cm}$ de altura, 89 individuos en el rango de 90 a $150 \mathrm{~cm}$ de altura, 202 individuos en el rango de 150 a $210 \mathrm{~cm}$ de altura, y 430 individuos tenían más de $2 \mathrm{~m}$ de altura. El porcentaje de iluminación promedio encontrado en los bosques evaluados es muy similar, aunque supera casi en un $6 \%$ a la cantidad de luz que llega al sotobosque de la selva tropical según lo indican los autores. Para evaluar la cantidad de iluminación promedio que llega al sotobosque, se tomaron, datos en 10 Carreteras Secundarias (CS) con Orientación Norte-Sur, 11 CS con orientación Este-Oeste, 12 Viales Principales (VP) con orientación Norte-Sur, 12 VP con orientación Este-Oeste, 12 Viales Secundarias (VS) con orientación Norte-Sur y 12 VS con orientación Este-Oeste. Además estas viales también se clasificaron por edad ya que, cuanto más joven fuera la vial (apertura más reciente) recibían un mayor porcentaje (\%) de iluminación en el sotobosque. El rango de años de apertura (edad) iba de 1 a 5 años de apertura.
\end{abstract}

Palabras clave: Bosque primario, iluminación, Uncaria tomentosa (Willd.) D.C, uña de gato, regeneración natural.

\footnotetext{
Abstract

The following research was carried out at the Alexander Von Humboldt National Forest BNAVH, belonging to the districts of Irazola, Tournavista and Puerto Inca in the provinces of Padre Abad and Puerto Inca at Huánuco and Ucayali regions respectively. The aim was to evaluate how climatic factors, specifically light, influence the natural regeneration of Uncaria tomentosa (Willd.) DC (Cat's Claw) in natural primary disturbed forests, located in the BNAVH. The evaluation was conducted in six forest types of primary forest characterized by the upper canopy opening caused by the effect of mechanized timber extraction and their orientation relative to the direction of solar illumination, inside which semi-permanent plots were established. In each one, Uncaria biological variables were measured with respect to how much light reached the understory. In these forest types more than 900 individuals of natural regeneration in good phytosanitary conditions can be found per ha, with average diameters of $14 \mathrm{~mm}$. Regarding height, 211 individuals ranged from 30 to $90 \mathrm{~cm} ; 89$ ranged from 90 to $150 \mathrm{~cm} ; 210$ ranged from 150 to $210 \mathrm{~cm}$ and 430 ranged over $2 \mathrm{~m}$. The average light percentage found in these forests is
} 
very similar, but exceeds by almost $6 \%$ the value reported by other authors for rainforests. To evaluate the average light percentage reaching the understory, data were taken in 10 Secondary Roads (CS) with North-South orientation, 11 CS with east-west, 12 Main Road (VP) with northsouth orientation, 12 VP with east-west, 12 High Road (VS) with north-south and 12 VS with eastwest. These roads were also classified by age since the younger ones got highest light percentages $(\%)$ in the understory. Road opening ages ranged from 1 year (youngest) to 5 years.

Key words: National Forest, lighting, Uncaria tomentosa (Willd.) DC, cat's claw, natural regeneration.

\section{Introducción.}

El Perú cuenta con 71.8 millones de hectáreas de bosques naturales, de los cuales el $92 \%$ constituyen los bosques húmedos tropicales (Reynel \& Alban, 1985; Zavala \& Zevallos, 1996), en donde se concentra una alta diversidad biológica por lo que se le considera como uno de los diez países "megadiversos" del mundo (Brack, 2000).

Dentro de los bosques tropicales, la cantidad de luz que penetra en la vegetación y puede llegar al suelo varía tanto por la densidad como por la posición de las hojas. En un bosque lluvioso tropical denso, por lo general, solo entre un 0.25 por ciento y un 2 por ciento de la luz que llega al dosel superior, atraviesa por completo la densa vegetación. En áreas de bosque más abierto, bosques intervenidos por ejemplo, la luz puede filtrarse más fácilmente (Chadzon \& Pearcy, 1991). En estos bosques es posible encontrar cinco capas o estratos principales, los cuales son más conspicuos en bosques inalterados. En algunos casos la estratificación suele estar poco definida, ya que el plan de crecimiento de muchas especies puede ocupar más de un estrato a la vez, como en el caso de las plantas epífitas, lianas y estranguladoras (Golley, 1983). Las lianas, por ejemplo pueden extenderse desde el suelo al dosel superior, volver a bajar y volver a trepar, alcanzando largas dimensiones. Ellas pueden crecer de manera prolífica en los claros del bosque (Denslow, 1987; Golley, 1983).

La estratificación de la vegetación en los bosques tropicales lluviosos, influye sobre el microclima interior del bosque y del porcentaje de luz que llega al sotobosque (que puede ser del 1 al $3 \%$ de lo que llega al dosel), la mitad llega al suelo en forma de salpicaduras de rayos solares; cerca de un $6 \%$ de la luz que alcanza el suelo lo hace directamente, gracias a ventanas abiertas en la bóveda, mientras que un $44 \%$ proviene de la luz reflejada y transmitida (Denslow, 1987; Golley, 1983; Lamprech, 1990). En los bosques tropicales lluviosos y montanos de la selva peruana, se pueden encontrar diferentes niveles de iluminación y temperatura, lo cual determina una alta diversidad vegetal que suele agruparse de acuerdo a los diferentes usos y/o servicios ambientales que brindan (Mercado, 2010). Un grupo importante de esta diversidad son las plantas medicinales y en la Amazonía peruana como una de las más importantes está Uncaria tomentosa (Willd) DC, llamada comúnmente "uña de gato". Esta especie nativa, es utilizada desde tiempos inmemoriales por diversos grupos étnicos de la selva en el tratamiento de sus diversas enfermedades. El conocimiento de este valioso recurso ha sido preservado por tradición oral de generación en generación, llegando sólo a trascender el campo de la medicina occidental en las últimas décadas (Flores, 1999; Domínguez, 1997; Zavala \& Zevallos, 1996; Quevedo \& Gil, 1998; Quinteros, 2001). Esta especie ha logrado ingresar de manera acelerada, al mercado nacional e internacional, dado que el $80 \%$ de la población mundial recurre a la medicina tradicional para atender sus necesidades primarias médicas, arriesgando su abastecimiento a futuro (Guerrero, 2001).

U. tomentosa (Willd) DC es una especie típica de bosques primarios y se desarrolla en los claros del bosque y en las capas superficiales del suelo, lo que sugiere que es relativamente exigente en nutrientes y en iluminación. También se le puede encontrar en bosques ligeramente intervenidos (Lombardi \& Zevallos, 1999), donde se encuentran plantas muy desarrollados y con diámetros considerables, posiblemente muy viejos, donde sólo hubo extracción selectiva de especies maderables comerciales. En estos ambientes la entrada de rayos solares hasta la superficie del suelo es escasa. Sin embargo esta condición hace que la planta se oriente hacia la luz y tome la forma de liana, estableciendo su follaje en la copa de los árboles, por lo que se le considera como una especie heliófita (Domínguez, 1997). La "uña de gato" se encuentra en lugares muy variados, desde terrenos inundados temporalmente, durante la creciente de los ríos amazónicos, hasta en terrenos altos y colinosos, de suelos fértiles, preferentemente orgánicos y de buen drenaje (Lombardi \& Zevallos, 1999).

La "uña de gato" naturalmente se encuentra asociada con los siguientes árboles: huayruro (Ormosia sp.), marupá (Simarouba amara), lupuna (Chorisia sp.), shihuahaco (Dypteris alata), tacho (Terminalia oblonga), cumala amarilla (Iriartea sp.), banderilla roja (Jacaranda sp.), cedro (Cedrela odorata), caoba (Swietenia macrophylla), roble amarillo (Terminalia tarapotensis), tornillo (Cedrelinga catenaeformis), capirona (Calycophyllum spruceanum), balata (Pouteria sp.), zapotillo blanco 
(Quararibea sp.), entre las más importantes (Lombardi \& Zevallos, 1999).

El objetivo principal de este trabajo fue estudiar las condiciones del hábitat natural de la especie, evaluando para ello el factor iluminación y cómo éste influye en el establecimiento y desarrollo de la regeneración natural de la Uncaria tomentosa (Willd.) D.C (Uña de gato), a fin de facilitar su manejo en bosques naturales para lograr un adecuado uso sostenible del recurso.

\section{Materiales y métodos. \\ Área de estudio}

El presente estudio de investigación fue llevado a cabo en el Bosque Nacional Alexander Von Humboldt- BNAVH, jurisdicción del distrito de Irazola, Provincia de Padre Abad y los distritos de Tournavista y Puerto Inca, Provincia de Puerto Inca, Regiones de Ucayali y Huánuco, respectivamente.

El área de trabajo se ubicó en el ámbito de 1500 ha de bosque primario del área total del BNAVH (469 $744,65 \mathrm{ha}$ ), que se enmarca aproximadamente entre las coordenadas geográficas de $8^{\circ} 25^{\prime}$ a $9^{\circ} 36^{\prime}$ LS y de $74^{\circ} 59^{\prime}$ a $75^{\circ} 30^{\prime}$ LO, como se puede observar en la Figura 1. Altitudinalmente ocupa elevaciones entre 250 a 500 msnm.

La evaluación, se realizó en diferentes tipos de bosque primario intervenido por la extracción mecanizada de la madera cerca de las parcelas abandonadas del Lote 3, del Ex - proyecto ITTO PD 95/90 (F): Plan de Manejo Forestal del Bosque Nacional Alexander Von Humboldt. La temperatura promedio es de $25^{\circ} \mathrm{C}$, siendo la temperatura máxima $35^{\circ} \mathrm{C}$ y la mínima $14^{\circ} \mathrm{C}$. Los meses más lluviosos son de octubre a marzo y los más secos de mayo a setiembre. El área donde se establecieron las parcelas para este estudio, pertenecía a la Zona de Vida clasificada como Bosque muy húmedo Pre-montano Tropical (bmh-PT). En el ámbito del BNAVH, se encuentra una densa red hidrográfica, constituida por 31 riachuelos y quebradas de reducido caudal para la navegación permanente. Los únicos ríos que se utilizan como vías de transporte por embarcaciones de pequeño calado, son el Aguaytía, el Sungaroyacu y el Pachitea.

Consideraciones para la ubicación de parcelas El proyecto ITTO PD 95/90 (F) Proyecto de Plan de Manejo Forestal del BNAVH diseñó la ejecución de extracciones selectivas de especies maderables, sobre una superficie de 105,000 ha, dividida en Unidades de Manejo Forestal (UMF) de 3500 ha, las cuales, mientras el proyecto estuvo en ejecución, fueron sometidas a procesos cuidadosos de extracción. Para tal fin, se estableció una red de caminos forestales con ciertas características para poder llevar a cabo un adecuado aprovechamiento de Bosque Nacional. La superficie seleccionada para llevar a cabo el proyecto, fue clasificada por lotes, del 1 al 5. La red de caminos

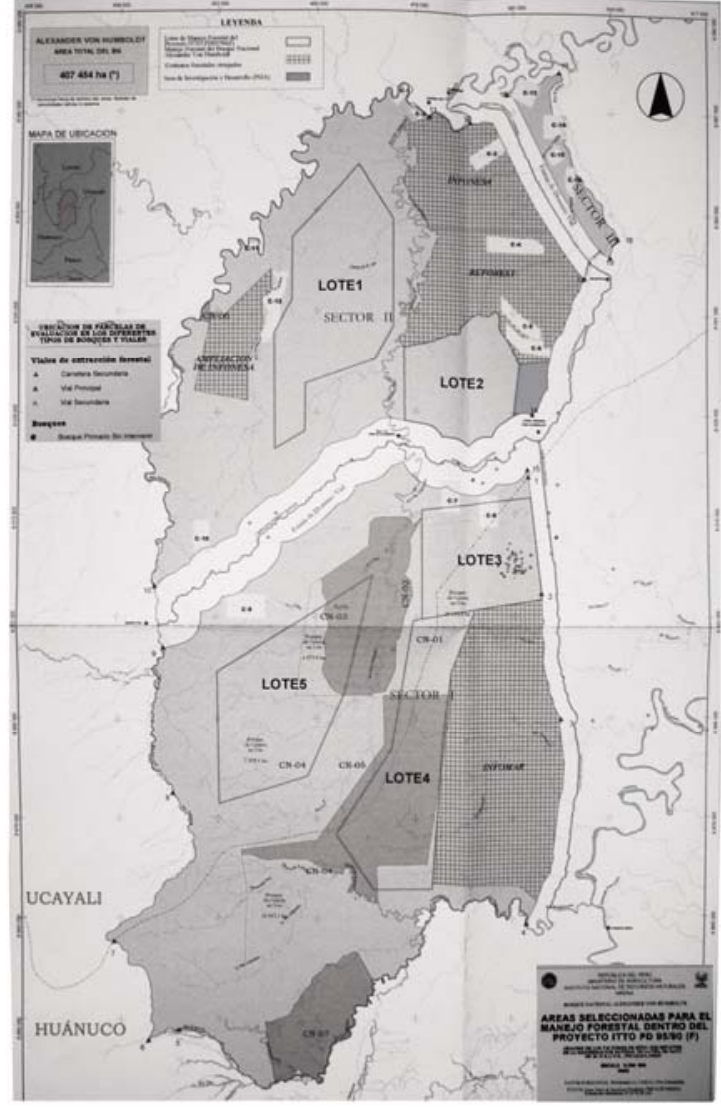

Figura 1. Mapa de Ubicación del Bosque Nacional Alexander Von Humboldt con sus diferentes Unidades de Gestión Forestal.

Fuente: Elaboración propia.

forestales servía para conectar precisamente estos lotes, a través de carreteras de acceso a cada lote, carreteras principales, carreteras secundarias y pistas de arrastre. Las especificaciones técnicas de estos caminos se presentan en la Tabla 1.

Tabla 1. Especificaciones técnicas para los caminos

\begin{tabular}{|c|c|c|c|}
\hline \multirow[b]{2}{*}{ ITEMS } & \multicolumn{3}{|c|}{ CLASE DE CAMINO } \\
\hline & $\begin{array}{c}\text { ACCESO } \\
\text { Y } \\
\text { PRINCIP } \\
\text { AL } \\
\end{array}$ & $\begin{array}{l}\text { SECUNDAR } \\
\text { IAS }\end{array}$ & $\begin{array}{c}\text { PISTAS DE } \\
\text { ARRASTRE } \\
\text { (Viales) }\end{array}$ \\
\hline Calidad $(\mathrm{Km} . / \mathrm{h})$ & 18 & 12 & 5 \\
\hline Ancho desmontado (m) & 16 & 8 & 3 \\
\hline Ancho de calzada (m) & 4 & 4 & 3 \\
\hline Ancho de la cuneta (m) & 1 & -- & -- \\
\hline $\begin{array}{l}\text { Radio mínimo curva } \\
\text { Horizontal }(\mathrm{m})\end{array}$ & 20 & 20 & -- \\
\hline Peralte & 5 & 5 & -- \\
\hline $\begin{array}{l}\text { Ensanches para cruce } \\
\text { (m) }\end{array}$ & $\mathrm{C} / 300$ & $\mathrm{C} / 400$ & -- \\
\hline Horizontal tierra suelta & 1 & 1 & -- \\
\hline Vertical tierra suelta & 1 & 1 & -- \\
\hline
\end{tabular}

Fuente: INRENA \& ITTO, 1998 Plan de Manejo Forestal del Bosque Nacional Alexander Von Humboldt. 
El trazado preliminar de las carreteras de acceso a cada lote y de las carreteras principales por lote presentan las longitudes estimadas en la Tabla 2.

Tabla 2. Longitudes de Carreteras de Acceso y Principales para el Aprovechamiento

\begin{tabular}{|c|c|c|c|c|}
\hline BNAVH & \multicolumn{3}{|c|}{ CARRETERA DE ACCESO (Km.) } & \multirow{2}{*}{$\begin{array}{l}\text { TOTAL } \\
(\mathrm{Km} .)\end{array}$} \\
\hline LOTE & $\begin{array}{l}\text { FEDERICO } \\
\text { BASADRE }\end{array}$ & $\begin{array}{l}\text { MARGINAL } \\
\text { DE LA SELVA }\end{array}$ & $\begin{array}{c}\text { CARRETERA } \\
\text { PRINCIPAL }\end{array}$ & \\
\hline 1 & 11.7 & 0 & 44.2 & 55.9 \\
\hline 2 & 3.9 & 0 & 31.2 & 35.1 \\
\hline 3 & -- & 5.5 & 31.6 & 37.2 \\
\hline 4 & -- & 0 & 45.5 & 45.5 \\
\hline 5 & 15.6 & 0 & 75.4 & 91.0 \\
\hline TOTAL & 31.2 & 5.6 & 227.9 & 264.7 \\
\hline
\end{tabular}

Fuente: INRENA \& ITTO, 1998 Plan de Manejo Forestal del Bosque Nacional Alexander Von Humboldt.

Las carreteras secundarias se trazaron sobre las divisorias de aguas y laderas de colinas, evitando en lo posible atravesar lechos de ríos.

Las especies a ser aprovechadas, fueron principalmente caoba (Swietenia macrophyla), ishpingo (Amburana cereaensis), tornillo (Cedrelinga cateniformis) y pumaquiro (Aspidosperma sp.), seleccionadas por su alto valor comercial. El ámbito de la presente investigación se encontraba en las inmediaciones de las Unidades de Manejo Forestal 1, 2 y 3. Las tres UMF señaladas estaban ubicadas en el Lote No. 3 del Ex - proyecto ITTO PD 95/90 (F).

La fisiografía de cada unidad de manejo forestal, de acuerdo a la estratificación fisiográfica realizada por el proyecto, estaba conformada por un paisaje colinoso, constituido por terrenos ondulados de contornos y pendientes suaves, hasta colinas bajas accidentadas, las mismas que se encontraban enmarcadas entre dos vertientes definidas, la del Mashihuaco y la del Macuya, las cuales están constituidas por una red de tributarios permanentes y quebradas encajonadas.

Los bosques señalados presentaban una composición florística muy heterogénea. Las especies más abundantes que caracterizan al bosque son: catahua (Hura crepitans), copaiba (Copaeifera sp.), estoraque (Myroxilon balsamun), lupuna (Ceiba pentandra), shihuahuaco (Dipterix odorata), ana caspi (Apuleia leiocarpa), camungo moena (Vochysia sp.) y maquisapa ñaccha (Apeiba aspera).

\section{Metodología de evaluación.}

Se establecieron parcelas de medición de $10 \times 10$ $\mathrm{m}$, es decir de $100 \mathrm{~m}^{2}$ o $0.01 \mathrm{ha}$, en lugares donde hubiera viales de extracción mecanizada de madera. Estas viales tenían de 1 a 5 años de descanso después de la última extracción de madera, (clasificadas por rango de edades). Cada parcela fue limpiada, señalizada, marcada y evaluada. El tamaño, número y ubicación de cada parcela siguió la Metodología para el Estudio de la Vegetación, propuesta por Matteucci et al. 1982.

Las parcelas de evaluación fueron establecidas en distintos tipos de bosque (Tabla 3).

En cada parcela establecida, se evaluó la regeneración natural de Uncaria tomentosa (Willd.) D.C que tuvieran aproximadamente entre 3 meses a 2 años de edad, es decir aquellos individuos que mostraran un hábito de crecimiento más parecido al de un arbusto joven que al de una liana.

\section{Parámetros ambientales evaluados}

Se evaluó la respuesta de la regeneración natural de la $U$. tomentosa (Willd.) D.C, frente al factor ambiental iluminación, tomando en consideración que la mayoría de los autores señalan a esta especie como

Tabla 3. Tipos de Bosque evaluados

\begin{tabular}{lc}
\hline \multicolumn{1}{c}{ Tipos de Bosque Primario Intervenido } & $\begin{array}{c}\text { Numero de } \\
\text { Parcelas }\end{array}$ \\
\hline * Carreteras Secundarias con Orientación Norte- & 10 \\
Sur & 11 \\
* Carreteras Secundarias con Orientación Este- & 12 \\
Oeste & 12 \\
* Viales Primarias con Orientación Norte- Sur & 12 \\
* Viales Primarias con Orientación Este- Oeste & 12 \\
* Viales Secundarias con Orientación Este- & Oeste \\
* Viales Secundarias con Orientación Este- & 12 \\
$\quad$ Oeste
\end{tabular}

heliófila, aunque dentro de estos registros no se señala que se comporte como una planta heliófita en todos los estadios de su crecimiento y desarrollo. Los datos de iluminación se obtuvieron analizando los siguientes parámetros:

Intensidad de luz que llega al sotobosque: Para obtener estos datos se usaron luxímetros de lectura directa, los datos se tomaron en lux, y se determinó el porcentaje de la cantidad de luz que recibe la regeneración natural del total de luz que llega al bosque, es decir con respecto al campo abierto o a la cantidad de luz que llega al dosel superior (copas de los árboles). Se tomó en cuenta que la intensidad de luz que llegaba al sotobosque, dependía de la cobertura arbórea que tuviera la parcela, según el tipo de bosque (vial o carretera) en que se encontraba. En la Tabla 4, se registra la clasificación de los datos por rangos de iluminación.

Orientación de las viales: Puesto que dependiendo de la orientación que tengan, la cantidad de luz (horas luz) que reciben generalmente es diferente, según experiencias de campo ya realizadas. Para determinar la orientación de la vial donde se ubicaba una parcela, 
se usó una brújula Suunto, una cinta métrica y un par de vigas.

Tabla 4. Rangos de iluminación (\%) que llegan hasta el sotobosque

\begin{tabular}{ccc}
\hline $\begin{array}{c}\text { Numeración para los } \\
\text { Intervalos de } \\
\text { iluminación }\end{array}$ & $\begin{array}{c}\text { \% Iluminación } \\
\text { sotobosque }\end{array}$ & $\begin{array}{c}\text { Etiquetas por } \\
\text { valor de } \\
\text { iluminación }\end{array}$ \\
\hline 1 & $1-10 \%$ & Muy poco \\
2 & $11-20 \%$ & Poco \\
3 & $21-30 \%$ & Moderado \\
4 & $31-40 \%$ & Iluminado \\
5 & $41-50 \%$ & Bien Iluminado \\
6 & $>50 \%$ & Muy iluminado \\
\hline
\end{tabular}

Tipo de Viales: Se consideró también para evaluar el nivel de iluminación que recibía el sotobosque, debido a que el ingreso de la luz (porcentaje de iluminación) variaba en función al tamaño y apertura de la vial, así en Viales Secundarias que eran más angostas, menos intervenidas tenían menos ingreso de luz a comparación de las Viales Principales, y éstas a su vez eran más angostas y tenían menos iluminación promedio que las Carreteras Secundarias.

Edad de las Viales: Se tomó en cuenta este factor puesto que dependiendo de la edad o antigüedad de las viales, el ingreso de la luz variaría. Entonces cuanto más antigua fuera la vial, es decir si tenía más tiempo de descanso, la cobertura arbórea sería mayor y el ingreso de luz sería menor. Al final ocurría que a mayor edad de las viales menor ingreso de luz, y a menor edad de la viales mayor ingreso de luz. Se tomaron en cuenta viales de 1, 2, 3, 4 y 5 años de antigüedad.

\section{Variables biológicas evaluadas}

Para evaluar la influencia del factor ambiental luz o iluminación, sobre la regeneración natural de $U$. tomentosa (Willd.) D.C, se midieron las siguientes características o variables biológicas

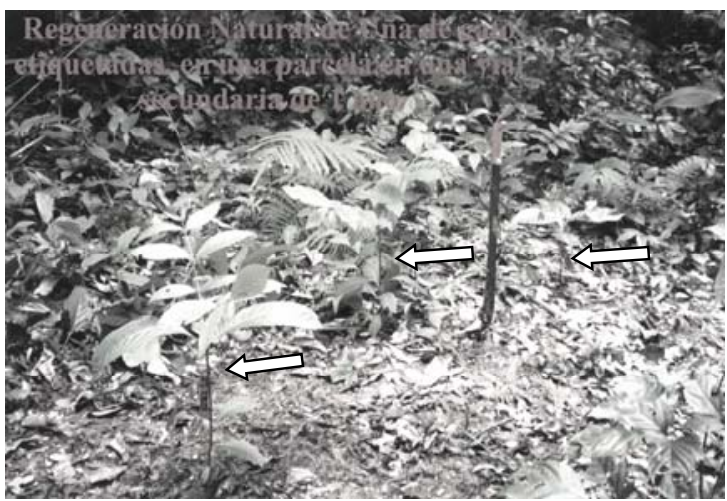

Figura 2. Densidad de la regeneración natural de la Uncaria tomentosa (Will) D.C.

a. Número de individuos por parcela (Densidad por parcela). En cada parcela de $100 \mathrm{~m}^{2}$, se hizo un conteo simple de individuos vivos presentes (Figura 2). De este conteo se calculó la densidad de individuos en el área total por cada tipo de vial en el Bosque Primario Intervenido (BPI).

b. Diámetro aproximado de la regeneración natural dentro de cada parcela. Para medir el diámetro promedio de cada uno de los individuos de la regeneración natural de $U$. tomentosa (Willd.) D.C, se usó un vernier. Se midieron todos los individuos hallados en todas las parcelas, a $30 \mathrm{~cm}$ de distancia del suelo.

c. Niveles de Vigor o Estado Fitosanitario de la regeneración natural de la Uña de gato dentro de cada parcela. Para determinar el vigor o estado fitosanitario de las plantas se tomaron los datos relacionados con estas características y se ordenaron por rangos de porcentajes (Tablas 5 y 6 ).

Tabla 5. Tipos y valores de vigor o estado fitosanitario de acuerdo al porcentaje de los diferentes tipos de ramas encontradas en los individuos de la regeneración natural de Uncaria tomentosa (Will) D.C.en cada parcela evaluada.

\begin{tabular}{ccc}
\hline Ramas Viejas & Ramas jóvenes & Puntaje de Vigor \\
\hline $0-34 \%$ & $>65 \%$ & 4 \\
$35-59 \%$ & $65-41 \%$ & 3 \\
$60-75 \%$ & $40-25 \%$ & 2 \\
$>75 \%$ & $<25 \%$ & 1 \\
\hline
\end{tabular}

Para poder manejar luego estas observaciones de manera cuantitativa se diseñó un sistema de valoración de vigor para cada parte observada en los individuos evaluados en el cual se ordena la información por presencia o ausencia de ramas viejas, ramas jóvenes o el estado fitosanitario de las hojas de cada uno de los individuos de $U$. tomentosa que fueran hallados en cada parcela trabajada (Tablas 5, 6 y 7).

Tabla 6. Tipos y valores de vigor o estado fitosanitario de acuerdo al porcentaje de las diferentes condiciones de las hojas de la regeneración natural de Uncaria tomentosa (Will) D.C. en cada parcela evaluada.

\begin{tabular}{cccccc}
\hline $\begin{array}{c}\text { Hojas } \\
\text { jóvenes }\end{array}$ & $\begin{array}{c}\text { Hojas } \\
\text { viejas }\end{array}$ & $\begin{array}{c}\text { Hojas } \\
\text { comidas }\end{array}$ & $\begin{array}{c}\text { Hojas con } \\
\text { hongos }\end{array}$ & $\begin{array}{c}\text { Hojas con } \\
\text { Manchas de } \\
\text { Necrosado }\end{array}$ & $\begin{array}{c}\text { Puntaje de } \\
\text { Vigor }\end{array}$ \\
\hline$>70 \%$ & $<30 \%$ & $0-20 \%$ & $0-20 \%$ & $0-20 \%$ & 4 \\
$50-70 \%$ & $30-50 \%$ & $21-50 \%$ & $21-50 \%$ & $21-50 \%$ & 3 \\
$30-50 \%$ & $50-70 \%$ & $51-80 \%$ & $51-80 \%$ & $51-80 \%$ & 2 \\
$<30 \%$ & $>70 \%$ & $>80 \%$ & $>80 \%$ & $>80 \%$ & 1 \\
\hline
\end{tabular}

De acuerdo con la Tabla 5, si un individuo de la regeneración natural de $U$. tomentosa (Will) D.C presentaba un 75 por ciento del total de ramas, en mal estado, es decir ramas marchitas o viejas, se consideraba que el estado fitosanitario o vigor de la planta, era bajo o pobre, por lo que el puntaje de vigor 
asignado a dicha planta sería de 1 o 2 . Bajo este mismo criterio se evaluaron las hojas de la regeneración natural (Tabla 6). Finalmente, se hizo un promedio del estado fitosanitario de cada planta de acuerdo a los valores obtenidos para sus hojas y sus ramas (Tabla 7).

Tabla 7. Valores totales y absolutos para determinar el vigor o estado fitosanitario de la regeneración natural de Uncaria tomentosa (Will) D.C.

\begin{tabular}{ccc}
\hline Estado Fitosanitario & $\begin{array}{c}\text { Valores } \\
\text { Absolutos }\end{array}$ & $\begin{array}{c}\text { Rangos de } \\
\text { Valores }\end{array}$ \\
\hline Vigoroso & 4 & $32-25$ \\
Medianamente vigoroso & 3 & $24-17$ \\
Poco vigoroso & 2 & $16-9$ \\
Enfermo/ Seco /Muriéndose & 1 & $8-1$ \\
\hline
\end{tabular}

d. Altura alcanzada por la reqeneración natural en cada parcela (Densidad de individuos según su altura)

Se midió la altura de cada individuo de cada parcela establecida dentro del (BPI) Bosque Primario Intervenido. Los datos obtenidos en estas mediciones se agruparon por rangos (clases de altura) según la longitud que alcanzaban $(30 \mathrm{~cm}, 90 \mathrm{~cm}, 1,50 \mathrm{~m}, 2,10$ $\mathrm{m}$ y más de $2,10 \mathrm{~m}$ ). Con dichos datos, se hicieron las pruebas estadísticas correspondientes que se presentan en los capítulos de Resultados y Discusión.

\section{Análisis Estadístico}

Para obtener una información lo más precisa posible de como afectaba o influía el factor iluminación sobre la regeneración natural de Uncaria tomentosa (Will) D.C. se sometieron los resultados de las variables biológicas y los parámetros ambientales a dos pruebas estadísticas específicas: una de correlaciones utilizando el Coeficiente de Correlación por Rangos de Spearman (Tabla 8) y otra de variancias, mediante el análisis de Kruskall-Wallis.

Tabla 8. Niveles de correlación dependiendo del coeficiente ro.

\begin{tabular}{cc}
\hline $\begin{array}{c}\text { Valor del coeficiente } \boldsymbol{r} \\
\text { (positivo o negativo) }\end{array}$ & Significado \\
\hline 0,00 a 0,19 & Correlación muy débil \\
0,20 a 0,39 & Correlación débil \\
0,40 a 0,69 & Correlación moderada \\
0,70 a 0,89 & Correlación fuerte \\
0,90 a 1,00 & Correlación muy fuerte \\
\hline
\end{tabular}

\section{Resultados y discusión.}

Los resultados obtenidos corresponden a los tipos de bosque analizados con sus respectivas variantes; para el caso de Bosque Primario Intervenido (BPI) por la extracción mecanizada de madera hay 6 tipos de viales por lo tanto 6 tipos de bosques primarios intervenidos, dentro de los cuales se evaluaron

Tabla 9. Porcentaje (\%) de iluminación promedio que llega al sotobosque de acuerdo al tipo de vial evaluada dentro del Bosque Primario Intervenido.

\begin{tabular}{lll}
\hline Iluminación por Tipo de Vial & $\begin{array}{c}\text { \% Iluminación } \\
\text { sotobosque }\end{array}$ & $\begin{array}{c}\text { Tipo de } \\
\text { iluminación }\end{array}$ \\
\hline Carretera Secundaria NS & $28 \%$ & Moderada \\
Carretera Secundaria EO & $26 \%$ & Moderada \\
Vial Principal NS & $22 \%$ & Moderada \\
Vial Principal EO & $23 \%$ & Moderada \\
Vial Secundaria NS & $29 \%$ & Moderada \\
Vial Secundaria EO & $19 \%$ & Poca \\
\hline
\end{tabular}

finalmente 69 parcelas.

En todos estos tipos de bosque se encontró regeneración natural de $U$. tomentosa, aunque en diferentes cantidades. Se determinó que el porcentaje de iluminación para los tipos de bosque es similar y aunque aparentemente es poco en relación a lo que llega a campo abierto, es lo previsto para este tipo de bosques tropicales intervenidos, según lo indican los diferentes autores consultados (Tablas 9 y 10) (Smith, 2004; Young, 1991; Jensen \& Salisbury, 1997; Lamprecht, 1990).

En general parece ser que la regeneración natural de $U$. tomentosa se establece y desarrolla mejor en las viales principales y secundarias del Bosque Primario Intervenido, y no así en las Carreteras Secundarias, a pesar que las condiciones de luz o iluminación en estas últimas eran mejores o de un mayor porcentaje de ingreso de la luz con respecto al dosel superior y al campo abierto (Tabla 11).

El diámetro y estado fitosanitario en cambio, tienen una correlación positiva pero muy débil con respecto a la iluminación, y solo para el caso del vigor de la planta, la prueba es estadísticamente significativa (Tabla 12). La altura alcanzada por los individuos evaluados, presenta correlaciones muy bajas y estadísticamente no significativas, respecto a la iluminación que llega al sotobosque, lo cual nos indica que este factor ambiental influye muy poco o nada en la altura alcanzada por la especie. Esto podría indicar que el factor iluminación, influye en la densidad y desarrollo de la planta pero, bajo ciertos rangos y a determinada edad, ya que es posible que si se superan los niveles de iluminación requeridos por la planta, el efecto llegaría a ser nulo o hasta negativo. Respecto a la orientación de las viales, se determinó que la regeneración natural de la $U$. tomentosa, puede establecerse indistintamente en viales con orientación Norte-Sur o Este-Oeste, pues sus variables biológicas muestran una correlación baja respecto a este parámetro. 
Tabla 10. Porcentaje (\%) de Iluminación que llega al Sotobosque de acuerdo al Tipo de Vial evaluada dentro del Bosque Primario Intervenido.

\begin{tabular}{lcl}
\hline $\begin{array}{c}\text { Iluminación por } \\
\text { Edad de la Vial }\end{array}$ & $\begin{array}{c}\% \\
\text { Iluminación } \\
\text { sotobosque }\end{array}$ & $\begin{array}{c}\text { Tipo de } \\
\text { iluminación }\end{array}$ \\
\hline Vial de 1 año & $38 \%$ & Iluminado \\
Vial de 2 años & $30 \%$ & Moderado \\
Vial de 3 años & $10 \%$ & Muy poco \\
Vial de 4 años & $27 \%$ & Moderado \\
Vial de 5 años & $19 \%$ & Poco \\
\hline
\end{tabular}

Análisis de Correlación dentro del Bosque Primario Intervenido (BPI)

Se observa que la densidad de la especie guarda una fuerte correlación positiva respecto al factor iluminación, aunque no es estadísticamente significativa.
La altura de la regeneración natural de la $U$. tomentosa presenta correlaciones débiles a moderadas, y estadísticamente significativas, frente a la edad de las viales, por lo que podemos suponer que cuanto más tiempo de descanso haya pasado desde la apertura de una vial, se podrán encontrar menos individuos jóvenes o regeneración natural, y esto puede deberse a la cantidad de iluminación que ingresa en viales más antiguas. Es posible, pero no determinante, porque el valor de la correlación es moderada a baja que, en las viales más antiguas o en ecosistemas boscosos con similares características de perturbación, solo se encuentren individuos de cierta altura, es decir de más de 1 metro de altura. Estos individuos podrían ser aquellos que se establecieron cuando la vial tenía menos tiempo de haber sido aperturada y permitía un mayor ingreso de luz.

Resultados estadísticos obtenidos de la prueba Kruskal-Wallis (K-S) para el Bosque Primario Intervenido (BPI)

Respecto al factor iluminación, sólo se obtuvo

Tabla 11. Variables biológicas evaluadas de la regeneración natural de la Uncaria tomentosa (Willd.) D.C dentro del Bosque Primario Intervenido del BNAVH.

\begin{tabular}{|c|c|c|c|c|c|c|}
\hline $\begin{array}{c}\text { Variables biológicas de acuerdo al tipo de } \\
\text { Bosque Secundario evaluado }\end{array}$ & $\begin{array}{l}\text { Carretera } \\
\text { Secundaria } \\
\text { Norte-Sur }\end{array}$ & $\begin{array}{l}\text { Carretera } \\
\text { Secundaria } \\
\text { Este-Oeste }\end{array}$ & $\begin{array}{c}\text { Vial } \\
\text { Principal } \\
\text { Norte- } \\
\text { Sur }\end{array}$ & $\begin{array}{c}\text { Vial } \\
\text { Principal } \\
\text { Este- } \\
\text { Oeste }\end{array}$ & $\begin{array}{c}\text { Vial } \\
\text { Secundaria } \\
\text { Norte-Sur }\end{array}$ & $\begin{array}{c}\text { Vial } \\
\text { Secundaria } \\
\text { Este-Oeste }\end{array}$ \\
\hline Densidad por hectárea & 1140 & 927 & 1050 & 2592 & 1367 & 958 \\
\hline Diámetro Promedio & 15.2 & 13.6 & 12.4 & 13.4 & 13 & 13.4 \\
\hline Valoración del Vigor & 24 & 22 & 19 & 22 & 19 & 21 \\
\hline Vigor general o Estado Fitosanitario & Mv & $\mathrm{Mv}$ & Mv & Mv & Mv & Mv \\
\hline № de Individuos con Alturas de $30-90 \mathrm{~cm}$. & 210 & 140 & 520 & 570 & 460 & 210 \\
\hline № de Individuos con Alturas de $90-150 \mathrm{~cm}$. & 90 & 90 & 120 & 300 & 190 & 100 \\
\hline № de Individuos con Alturas de $150-210 \mathrm{~cm}$. & 260 & 320 & 120 & 850 & 280 & 190 \\
\hline № de Individuos con Alturas de + de $210 \mathrm{~cm}$. & 580 & 470 & 500 & 1390 & 710 & 650 \\
\hline
\end{tabular}

$\mathrm{Mv}=$ Medianamente vigoroso (Estado Fitosanitario de la regeneración natural)

También se encontró que las variables biológicas de densidad, diámetro, vigor y altura de la regeneración natural muestran una correlación muy baja respecto al tipo de vial en la cual se establece.

En cuanto a la correlación existente entre la densidad y vigor y la edad de las viales, se observa que no es muy fuerte, es negativa y estadísticamente significativo. Esto podría indicarnos que probablemente las condiciones de ecosistemas boscosos con las características de iluminación de las viales más jóvenes, tendrán un efecto positivo en el aumento de la densidad y el vigor de la regeneración natural de la $U$. tomentosa. Respecto al diámetro, según los resultados del cuadro, vemos que presenta una correlación moderada y estadísticamente significativa, con la edad de la vial, por lo que es muy probable que se encuentren individuos de mayor diámetro en viales con más edad o tiempo de descanso puesto que cuanto más antigua es la vial, la edad de la regeneración natural podría ser mayor o los individuos medidos son los que sobrevivieron a la primera etapa de establecimiento en el sotobosque. densidades significativamente más altas de los individuos de la regeneración natural de $U$. tomentosa que alcanzaban tamaños de entre 150 a $210 \mathrm{~cm}$ de altura, es decir de más de un año de edad, bajo condiciones de muy poca iluminación (Tabla 13). El resto de las variables o parámetros biológicos evaluados no presentan diferencias significativas en función al porcentaje de iluminación que llega al sotobosque y por lo tanto a la regeneración natural de esta especie. Estos resultados indican que la influencia del factor iluminación, por sí sólo, no es del todo determinante para la germinación y establecimiento de la regeneración natural.

El factor orientación de la vial, (factor relacionado no solo con el porcentaje de luz que llega al sotobosque sino con las horas de luz diarias) no parece ser determinante o que influya significativamente en el establecimiento y desarrollo de $U$. tomentosa, desde que germina hasta que deja de ser regeneración natural. 
Tabla 12: Índices de correlación entre el factor Iluminación y las variables biológicas de la regeneración natural de la Uncaria tomentosa (Willd.) D.C.

\begin{tabular}{|c|c|c|c|c|c|}
\hline Parámetros Biológicos & Prueba correlación & $\begin{array}{l}\text { Iluminación } \\
\text { sotobosque\% }\end{array}$ & $\begin{array}{l}\text { Orientación } \\
\text { de Vial }\end{array}$ & $\begin{array}{l}\text { Tipo } \\
\text { de } \\
\text { Vial }\end{array}$ & $\begin{array}{c}\text { Etapa } \\
\text { sucesional o } \\
\text { Edad de la Vial }\end{array}$ \\
\hline \multirow{2}{*}{ Densidad (№ Indv/100m2) } & $r_{\mathrm{o}}$ & 0.92 & -0.015 & -0.08 & -0.31 \\
\hline & Nivel de significancia & 0.46 & 0.9 & 0.5 & 0.01 \\
\hline \multirow{2}{*}{ Diámetro Promedio } & $r_{0}$ & 0.09 & 0.03 & 0.06 & 0.54 \\
\hline & Nivel de significancia & 0.46 & 0.84 & 0.6 & 0.0001 \\
\hline \multirow{2}{*}{ Vigor General } & $r_{\mathrm{o}}$ & 0.33 & 0.03 & 0.2 & -0.38 \\
\hline & Nivel de significancia & 0.01 & 0.77 & 0.1 & 0.001 \\
\hline \multirow{2}{*}{ № Indv con una altura de $30-90 \mathrm{~cm}$. } & $r_{\mathrm{o}}$ & 0.15 & -0.006 & 0.12 & -0.51 \\
\hline & Nivel de significancia & 0.22 & 0.96 & 0.34 & 0.0001 \\
\hline \multirow{2}{*}{ № Indv con una altura de $90-150 \mathrm{~cm}$. } & $r_{\mathrm{o}}$ & 0.002 & -0.05 & 0.05 & -0.3 \\
\hline & Nivel de significancia & 0.99 & 0.7 & 0.7 & 0.01 \\
\hline \multirow{2}{*}{ № Indv con una altura de $150-210 \mathrm{~cm}$. } & $r_{\mathrm{o}}$ & -0.17 & 0.05 & 0.22 & -0.22 \\
\hline & Nivel de significancia & 0.16 & 0.7 & 0.06 & 0.07 \\
\hline \multirow{2}{*}{ № Indv con una altura de + de $210 \mathrm{~cm}$. } & $r_{\mathrm{o}}$ & -0.2 & 0.034 & $\begin{array}{c}- \\
0.014\end{array}$ & 0.33 \\
\hline & Nivel de significancia & 0.1 & 0.8 & 0.9 & 0.01 \\
\hline
\end{tabular}

$N=69 \quad N$ : Número total de parcelas evaluadas en el Bosque Primario Intervenido (BPI) dentro del BNAVH

Sin embargo, podría existir una mayor preferencia o mejor respuesta en cuanto a una mayor densidad de individuos en viales que tengan una orientación EsteOeste o en ecosistemas boscosos que presenten el mismo nivel de iluminación que estas viales.

Según el tipo de vial en la cual se establezca la regeneración natural de $U$. tomentosa, vemos que generan un tipo de respuesta que no varía significativamente, entre una y otra, pero si podemos observar mayores valores de las variables biológicas evaluadas en viales principales, por lo que podríamos decir que la especie se establecerá mejor en ecosistemas boscosos con características similares de iluminación a las presentadas en las VP del BNAVH.

Una mención especial merece el factor edad de las viales puesto que en el análisis anterior de correlaciones, todos los valores obtenidos para $U$. tomentosa fueron estadísticamente significativos

Tabla 13 Prueba Kruskal-Wallis (K-S) aplicada a los parámetros del factor Iluminación y las variables biológicas de la regeneración natural de la Uncaria tomentosa (Willd.) D.C, evaluadas en los bosques primarios intervenidos (BPI) dentro del BNAVH.

\begin{tabular}{|c|c|c|c|c|c|c|c|c|c|}
\hline \multicolumn{2}{|c|}{ Factor Ambiental Iluminación } & $\mathrm{N}$ & $\begin{array}{c}\text { Densidad } \\
\left(\mathrm{N}^{\circ} \text { Indv } / 100 \mathrm{~m}^{2}\right)\end{array}$ & $\begin{array}{l}\text { Diámetro en } \mathrm{mm} \\
\text { (a } 30 \mathrm{~cm} . \text { del suelo) }\end{array}$ & $\begin{array}{c}\text { Vigor o } \\
\text { Estado } \\
\text { Fitosanitario }\end{array}$ & \multicolumn{4}{|c|}{$\begin{array}{c}\text { Densidad ( } \mathrm{N}^{\circ} \text { Indv/100m2) de acuerdo a su rango } \\
\text { de Altura }\end{array}$} \\
\hline \multirow{5}{*}{$\begin{array}{l}\% \text { de } \\
\text { Iluminación }\end{array}$} & 1 a $10 \%$ & 11 & $25 \pm 45.22$ & $12.77 \pm 3.57$ & $3 \pm 0.79$ & $3 \pm 4.59$ & $3 \pm 5.28$ & $9 \pm 16.68$ & $\begin{array}{l}10 \pm \\
19.85\end{array}$ \\
\hline & 11 a $20 \%$ & 20 & $12 \pm 9.75$ & $14.34 \pm 3.49$ & $3 \pm 0.64$ & $1 \pm 1.64$ & $1 \pm 1.57$ & $3 \pm 3.59$ & $8 \pm 4.22$ \\
\hline & 21 a $30 \%$ & 12 & $9 \pm 4.66$ & $13.96 \pm 3.61$ & $3 \pm 0.87$ & $1 \pm 1.27$ & $1 \pm 0.65$ & $1 \pm 0.75$ & $5 \pm 4.81$ \\
\hline & 31 a $40 \%$ & 14 & $9 \pm 7.64$ & $15.2 \pm 7.36$ & $4 \pm 0.63$ & $4 \pm 8.36$ & $1 \pm 1.01$ & $1 \pm 1.14$ & $4 \pm 3.73$ \\
\hline & Estimado estadístico & $\mathrm{P}$ & $>0.05$ & $>0.05$ & $>0.05$ & $>0.05$ & $>0.05$ & $<0.05$ & $>0.05$ \\
\hline \multirow{3}{*}{$\begin{array}{l}\text { Orientación } \\
\text { de la Vial }\end{array}$} & Norte - Sur & 34 & $12 \pm 9.31$ & $13.43 \pm 5.4$ & $3 \pm 0.77$ & $4 \pm 7.78$ & $1 \pm 1.73$ & $2 \pm 2.62$ & \multirow{2}{*}{$\begin{array}{l}5 \pm 4.29 \\
7 \pm \\
11.59\end{array}$} \\
\hline & Este - Oeste & 35 & $15 \pm 26.3$ & $13.47 \pm 4.79$ & $3 \pm 0.74$ & $3 \pm 5.42$ & $1 \pm 3.19$ & $4 \pm 9.86$ & \\
\hline & Estimado estadístico & $\mathrm{P}$ & $>0.05$ & $>0.05$ & $>0.05$ & $>0.05$ & $>0.05$ & $>0.05$ & $>0.05$ \\
\hline \multirow{6}{*}{$\begin{array}{l}\text { Edad de la } \\
\quad \text { Vial }\end{array}$} & 1 año & 8 & $17 \pm 11.9$ & $5.56 \pm 3.11$ & $4 \pm 0.01$ & $\begin{array}{l}15 \pm \\
13.72\end{array}$ & $1 \pm 0.53$ & $1 \pm 0.46$ & $1 \pm 1.07$ \\
\hline & 2 años & 16 & $16 \pm 7$ & $11.51 \pm 2.61$ & $3 \pm 0.73$ & $3 \pm 2.6$ & $2 \pm 2$ & $5 \pm 2.91$ & \multirow{5}{*}{$\begin{array}{l}6 \pm 3.71 \\
4 \pm 5.19 \\
6 \pm 3.91 \\
10 \pm \\
16.39 \\
<0.05\end{array}$} \\
\hline & 3 años & 4 & $5 \pm 4.03$ & $13.35 \pm 2.75$ & $3 \pm 0.82$ & $1 \pm 0.96$ & $1 \pm 0.5$ & $3 \pm 1.5$ & \\
\hline & 4 años & 25 & $9 \pm 5.34$ & $16.1 \pm 4.28$ & $3 \pm 0.69$ & $1 \pm 1.8$ & $1 \pm 1.08$ & $1 \pm 1.73$ & \\
\hline & 5 años & 16 & $19 \pm 38.91$ & $15.41 \pm 4.54$ & $2.7 \pm 0.7$ & $1 \pm 3.69$ & $2 \pm 4.62$ & $5 \pm 14.52$ & \\
\hline & Estimado estadístico & $\mathrm{P}$ & $<0.05$ & $<0.05$ & $<0.05$ & $<0.05$ & $<0.05$ & $<0.05$ & \\
\hline
\end{tabular}


En la prueba de K-S, obtuvimos densidades mayores, tanto en viales muy jóvenes (tiempo de descanso de 1 a 2 años), como en viales antiguas (tiempo de descanso más de 4 años) pero se debe distinguir que en viales más jóvenes la densidad de individuos jóvenes o de menor altura es mayor, y todo lo contrario ocurre en viales más antiguas, pues en ellas se presentan individuos más altos y posiblemente con mayor tiempo de establecimiento (regeneración natural más antigua o mayor).

También el diámetro de los individuos es menor en viales más jóvenes o recientemente dejadas en descanso, a pesar de tener más luz que aquellas viales que tiene más tiempo de descanso, son más antiguas, y por ende reciben menor cantidad de luz en el sotobosque pero es posible que los individuos sean mayores también. En cuanto al estado fitosanitario de las plantas, estas presentan individuos más vigorosos en viales más jóvenes.

Todo esto implica que, esta especie necesita un ambiente recientemente intervenido, con una iluminación promedio o buena, pero sólo al principio para su germinación y establecimiento inicial. Es posible que la interacción de este factor (tipo de vial) con algún otro como la humedad, temperatura, o sustrato, es decir una acción conjunta entre la iluminación y los demás factores indicados, genere un efecto sinérgico sobre las variables biológicas de la regeneración natural de esta especie.

Es importante recordar que cuanto mayor sea el diámetro de los individuos que se encuentran bajo las condiciones de estos bosques tropicales, menor será su densidad y viceversa, por varias razones, de las cuales la más importante es "la competencia inter e intraespecífica".

\section{Conclusiones.}

1. La regeneración natural de Uncaria tomentosa (Willd.) D.C. se encuentran influenciada para su desarrollo y prendimiento en el campo por la cantidad de luz que llega al sotobosque, sin ser este factor el único, ni tampoco el limitante o determinante para que la regeneración natural desarrolle con gran éxito.

2. Este estudio muestra que el factor iluminación es más importante para los individuos más jóvenes y pequeños, pues les posibilita desarrollar mejor hasta que alcanzan determinada altura, lo cual puede estar relacionado también con su edad, cambios en sus procesos metabólicos, de respiración y fotosíntesis, su nivel de enraizamiento y competencia con las otras especies silvestres junto a las cuales crece.

3. La regeneración natural de $U$. tomentosa, presenta un fototropismo positivo. El porcentaje de iluminación no tiene que ser excesivo o abundante, ya que al tener hábito de liana, su crecimiento se ve orientado hacia donde exista una entrada de luz, por ejemplo una apertura en el dosel superior del bosque.

4. Aunque no se evaluó niveles de humedad, en los ecosistemas de los BPI evaluados, es posible que también este factor junto con el de iluminación, contribuyan, al prendimiento y establecimiento de la regeneración natural de U. tomentosa.

5. Si los individuos de la regeneración natural de la uña de gato se establecen de manera silvestre en ecosistemas boscosos intervenidos antrópicamente, sin tratamientos o cuidados especiales y además, pueden llegar a presentan un desarrollo óptimo de su estado fitosanitario, diámetro, altura y densidad, precisamente en esta etapa pre juvenil en que, la mayoría de las especies requieren más cuidado y dedicación, significaría entonces que, trabajar en su aprovechamiento y manejo sostenible, podría ser considerado como una verdadera alternativa económica para la población nativa y local de la selva peruana en donde esta especie se encuentra y distribuye.

\section{Agradecimientos.}

Los autores expresan su agradecimiento a los M. Sc. Mercedes Flores, M. Sc. Raúl Eyzaguirre, M. Sc. Juan Torres y la Dra. Consuelo Arellano y el Dr. Carlos Reynel, docentes de la Universidad Nacional Agraria "La Molina", por su orientación en la realización de la presente investigación así como en la revisión de la información recopilada en campo y en la revisión final del documento redactado. De igual manera al INIA de Pucallpa, el ICRAF de Pucallpa, la DRAU, el INRENA de Pucallpa y Lima y a la UNU, instituciones que brindaron su apoyo técnico, logístico, y en equipos, para la toma de datos para el trabajo de investigación. De igual forma agradecer al señor Teodoro Reyes y su hijo Litson, por brindar su invalorable conocimiento tradicional del bosque, y de los trabajadores de las instalaciones del Ex Proyecto ITTO, José, Abel y Miguel, sin los cuales la realización del arduo trabajo de campo no hubiera sido posible.

\section{Literatura citada.}

Arce J. 1996. Manejo de Bosques para Producción sostenible de Uña de gato - La experiencia del Valle del Palcazú. Resúmenes del Curso de Identificación, producción, propagación y manejo de la "Uña de gato"; organizado por la Facultad de Ciencias Forestales y llevado a cabo del 13 al 15 de Noviembre de 1996, en la Universidad Nacional Agraria "La Molina".

Barriga R. 1994. Plantas útiles de la amazonía peruana; características, usos y posibilidades.. CONCYTEC. Lima-Perú. 261 Pág.

Brack A. 2000. Ecología del Perú. Editorial Bruño. LimaPerú. 495 pág. 
Braingbridge R.; Evans G.C.; Rackham O. 1966. Light as an ecological factor Oxford, England: Blackwell Scientific publications.

Castillo A. 1993. Estudio de la Regeneración Natural de Cinco Especies Forestales en el Bosque Nacional Alexander Von Humboldt. Pucallpa-Perú. Tesis para optar el Grado de Magíster Scientiae. Escuela de PostGrado, Especialidad de Manejo Forestal. Universidad Nacional Agraria "La Molina”. Lima-Perú. 156 Pág.

Chadzon R.L. \& Pearcy R.W. 1991. The importance of sunflecks for forest understory plants. Bioscience 41: $760-765$.

Denslow J.S. 1987. Tropical Rainforest Gaps and Tree Species Diversity. Annual Review of Ecology and Systematics. Vol. 18: 431-451

Domínguez G. 1997. Uña de gato y producción sostenible. Publifor-Universidad Nacional Agraria "La Molina"FCF. Lima - Perú. 138 Pág.

Dourojeani M. \& Ferreyra R. 1986. Flora y Vegetación del Perú. Enciclopedia Gran Geografía del Perú. Tomo II. Manfer-Juan Megía Baca. Barcelona - España. 323

Dourojeani M. \& Brack A. 1986. Ecología de un País Complejo. Enciclopedia Gran Geografía del Perú. Tomo II. Manfer-Juan Megía Baca. Barcelona - España. 323

Estrada Z. 1993. Comportamiento de la Regeneración Natural de Especies Arbóreas en Diversos Tipos de Pasturas en la Zona de Pucallpa. Tesis para optar el título de Ingeniero Forestal. Facultad de Ciencias Forestales. Universidad Nacional Agraria "La Molina". 124 Pág.

Estrella E. 1995. Biodiversidad y salud en las Poblaciones Indígenas de la Amazonía. Tratado de Cooperación Amazónico - FAO - PNUD - Banco Mundial - Unión Europea- MINISTERIO DE COOPERACIÓN TÉCNICA DEL REINO DE LOS PAÍSES BAJOS UNAMAZ. Secretaría Pro Tempore Quito - Ecuador. 312 Pág.

Flores Y. 1999. Estudio Experimental del Crecimiento de Uña de Gato en Plantaciones Artificiales. Programa Nacional de Investigación en Agroforestería y Cultivos Tropicales. Instituto Nacional de Investigación Agraria INIA. Pucallpa - Perú. 20 Pág.

Fowler J. \& Cohen L. 1999. Estadística Básica en ornitología. Editorial: SEO/BIRDLIFE. ISBN: 9788492190164. España.144 págs.

Golley F.B. (ed) 1983. Tropical rain forest ecosystems: Structure and function Ecosystems of the world. Amsterdam. Elsevier Scientific Pub. Co. No. 14.A. 381 Pág

Guerrero A. 2001. Lineamientos y Estructura para el Diseño de la Estrategia Nacional Forestal. Documento de Trabajo No. 9. 2001. Proyecto FAO GCP/PER/035/NET. Apoyo a la Estrategia para el Desarrollo Forestal. Lima - Perú. 142 Pág.

INADE \& APODESA.1987. Experiencias Silviculturales y de Manejo de Bosques en América Latina. Seminario Taller llevado a cabo del 3 al 21 de Agosto de 1987. Compendio de las Presentaciones. OEA - USAID JICA - COTEBE - GTZ - ACDI. Lima-Perú. 270 Pág.

Instituto Nacional de Recursos Naturales INRENA. 1998. Proyecto ITTO PD 95/90 (F) Manejo Forestal del Bosque Nacional Alexander Von Humboldt. Plan de Manejo Forestal del Bosque Nacional Alexander Von Humboldt. INR - 86 - DGF. Lima - Perú. 98 Pág.
Instituto Nacional de Recursos Naturales INRENA. 1995. Guía Explicativa del Mapa Forestal. INR - 49 - DGF. Lima-Perú. 131 Pág.

Instituto Nacional de Recursos Naturales INRENA. 1995. Mapa Ecológico del Perú. Guía Explicativa y Mapa. Ministerio de Agricultura. Lima, Perú.

Jensen W. \& Salisbury F. 1997. Botánica. Segunda edición (Primera edición en español). McGRAW-HILL. México - México. 762 Pág.

Lamprecht H. 1990. Silvicultura en los Trópicos, los ecosistemas forestales en los bosques tropicales y sus especies arbóreas, posibilidades y métodos para un aprovechamiento sostenido. Instituto de Silvicultura de la Universidad de Göttingen. Cooperación TécnicaRepública Federal de Alemania. 335 Pág.

Lock O. 1994. Investigación Fitoquímica. Métodos en el estudio de productos naturales. Pontificia Universidad Católica del Perú. Fondo Editorial. Lima - Perú. 300 Pág.

Lombardi I. \& Zevallos P. 1999. Guía para el cultivo, aprovechamiento y conservación de la Uña de Gato, Uncaria tomentosa (Willd. ex Roemer \& Schultes) De Candolle. CAB - SECAB. Santa Feb de Bogotá Colombia.19 Pág.

Malleaux J. 1982. Inventarios Forestales en Bosques Tropicales. UNALM, OEA. Lima - Perú. 414 Pág.

Matteucci S.; Colma A.; Frangi J.L. 1982. Metodología para el Estudio de la Vegetación. CONICIT, OEA Programa Regional de Desarrollo Científico y Tecnológico. Washington, D.C. - EE.UU. 163 Pág

Mejía K. \& Rengifo E. 1995. Plantas Medicinales de Uso Popular en la Amazonía Peruana. AECI-GFL-HAPIIAP. Lima-Perú. 284 Pág.

Mercado W. 2010. Negocios Rurales. La economía de la biodiversidad y los bionegocios. Revista Agronegocios. Facultad de Economía y Planificación de la Universidad Nacional Agraria La Molina - UNALM. Lima, Perú. Año 4 - N 3. Pág. 3-5.

Nebel G.; Kvist L.P.; Vanclay J.; Vidaurre H. 2000. Dinámica de los bosques de la llanura aluvial inundable de la Amazonía Peruana; efecto de las perturbaciones e implicancias para su manejo y conservación. Folia Amazónica, Vol. 11, No 1-2. Instituto de Investigaciones de la Amazonía Peruana - IIAP. Iquitos - Perú. Pág. 6597.

Nebel G. 2000. Estructura y composición florística del bosque de la llanura aluvial inundable de la Amazonía Peruana: I. El bosque alto. Folia Amazónica, Vol. 10, N 1-2. Instituto de Investigaciones de la Amazonía Peruana - IIAP. Iquitos - Perú. Pág. 91-149.

OIMT. 1999. Manual sobre la Aplicación de Criterios e Indicadores para la Ordenación sostenible de los Bosques Tropicales Naturales. Parte A / Indicadores a Nivel Nacional. OIMT- Serie de políticas forestales $\mathrm{N}^{\circ}$ 9.1999. Yokohama. Japón. 52 Pág.

OIMT. 1999. Manual sobre la Aplicación de Criterios e Indicadores para la Ordenación sostenible de los Bosques Tropicales Naturales. Parte B / Indicadores a Nivel de la unidad de Ordenación Forestal. OIMT- Serie de políticas forestales $\mathrm{N}^{\circ} 10.1999$. Yokohama. Japón. 48 Pág.

Pyhälä M.; Salo J.; Kalliola R.; Padoch C.; Pinedo M.; Torres J. 2001. Amazonía: Orientaciones para el 
Desarrollo Sostenible. Embajada de Finlandia. UNAP, BCRP-Fondo Editorial, PNUD. Lima-Perú.170 Pág.

Quevedo A. \& Gil O. 1998. Efecto de la intensidad de la luz, método de conservación y tiempo de almacenamiento en la germinación de Croton lecheri Muell. Arg. Folia Amazónica, Vol. 9, $\mathrm{N}^{\mathrm{o}}$ 1-2. Instituto de Investigaciones de la Amazonía Peruana - IIAP. Iquitos - Perú. Pág. 4561.

Quinteros B. 2001. Distribución Natural y Determinación Edafoclimática de la Uncaria tomentosa (Wild.). D.C. y Uncaria guianensis (Aubl.) Gmel (Uña de gato), en la cuenca del río Aguaytía. Tesis para optar e título de Ingeniero Forestal. Universidad Nacional de Ucayali. Facultad de Ciencias Forestales. Pucallpa - Perú. 86 Pág.

Reynel C. \& Albán J. 1985. Cuatro especies forestales con potencialidad alimenticia en la amazonía peruana. Rev. For. Perú 13: 60-79
Smith R. \& Smith T. 2004 ECOLOGÍA. Cuarta Edición. PEARSON EDUCATION S.A.; Addison - Wesley. Madrid, España. 664 Pág.

Weberbauer A. 1945. El mundo vegetal de los Andes peruanos. Estudio fitogeográfico. (Nueva edición, revisada y ampliada de: Die Pflanzenwelt der peruanischen Anden, Leipzig 1911), 776 S., Lima: Ministerio de Agricultura.

Young R. \& Hurtado J. 1991. Introducción a la Ciencias Forestales. Editorial LIMUSA S. A. de C. V. México D. F. - México. 637 Pág.

Zavala C. \& Zevallos P. 1996. Taxonomía, distribución geográfica y status del Género Uncaria en el Perú - Uña de gato. INDDA-Universidad Nacional Agraria "La Molina". Lima-Perú. 106 Pág.

\footnotetext{
${ }^{1}$ Universidad Nacional Agraria La Molina, Av La Molina s/n, La Molina, Lima Perú. awetzell@lamolina.edu.pe, awetzell@gmail.com, awetzellcs@yahoo.es

2 Universidad Nacional Agraria La Molina, Av La Molina s/n, La Molina, Lima Perú. aceroni@lamolina.edu.pe

3 Universidad Nacional Agraria La Molina, Av La Molina s/n, La Molina, Lima Perú. gdominguez@lamolina.edu.pe

4 Anterior: Instituto de Investigación de la Amazonia Peruana, Pucallpa, Perú. Actual: Universidad Nacional Intercultural de la Amazonia Peruana - Yarinacocha. Pucallpa, Ucayali Perú. acastillo_2519@hotmail.com
} 